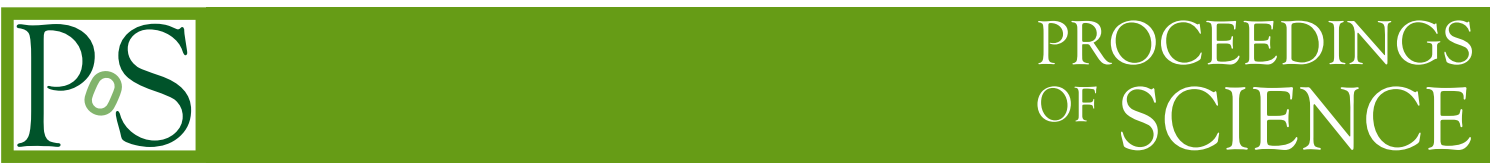

\title{
Measurements with hadron beams at COMPASS
}

\author{
Andrea Ferrero* \\ Dipartimento di Fisica Generale "A. Avogadro" and INFN Torino, Italy \\ E-mail: aferrero@to.infn.it
}

\section{On behalf of the COMPASS Collaboration}

The physics program of the COMPASS experiment at CERN focuses on the investigation of the hadron structure and spectroscopy, using both leptonic and hadronic probes. The COMPASS experiment has collected so far mostly data with polarized muon beams of $160 \mathrm{GeV}$, but also a pilot data taking with negative hadron beams of $190 \mathrm{GeV}$ has been successfully completed at the end of the 2004 run.

The main physics objectives of this pilot run are the study of soft pion-nuclei interactions. The predictions of the Chiral Perturbation Theory for the electric and magnetic polarizabilities of the pion will be verified through the study of the Primakoff scattering of $190 \mathrm{GeV}$ pions on thin lead targets. A sample corresponding to an integrated beam flux of more than $10^{11}$ pions has been collected for this purpose, and an equivalent sample with the muon beam of $190 \mathrm{GeV}$ has been collected in the same experimental conditions to correct possible systematic effects.

In parallel to the polarizability measurements, first data have also been collected for the study of the diffractive production of exotic non- $q \bar{q}$ states. Such states, although predicted by QCD, still require a confirmation from the experimental side. This measurements are part of a wider research program, which includes the study of gluon-rich states produced in central proton-proton collisions, that will be addressed by the COMPASS experiment in the forthcoming years.

The performances of the apparatus during the pilot COMPASS run with hadron beams will be presented, and the preliminary results of the analysis of the collected data will be discussed. The future perspectives of the proposed measurements will also be briefly outlined.

International Europhysics Conference on High Energy Physics

July 21st - 27th 2005

Lisboa, Portugal

${ }^{*}$ Speaker. 


\section{Introduction}

COMPASS is a fixed target experiment installed at the SPS accelerator complex of CERN, aiming at the study of the structure and spectroscopy of hadrons, using both leptonic and hadronic probes. An important part of the COMPASS physics program is the study of non-conventional hadronic states with valence gluons, that can be either hybrid mesons $(q \bar{q} g)$ or glueballs $(g g)$, and are characterized by spin, parity and charge conjugation quantum numbers forbidden by $q \bar{q}$ states. These exotic states are identified from the Partial Wave Analysis (PWA) of the resonant states produced in peripheral pion-nucleus interactions (diffractive scattering and photoproduction) and in central proton-proton collisions. Calculations performed in the framework of the flux-tube model $[1,2]$ predict a mass of about $1.9 \mathrm{GeV}$ for the lightest hybrid meson state $\left(J^{P C}=1^{-+}\right)$, that is expected to decay in $\eta \pi, \eta^{\prime} \pi, \rho \pi, f_{1} \pi$ and $b_{1} \pi[3]$. Several hybrid candidates have been reported by different collaborations $[4,5,6,7,8,9,10]$ in the $\eta \pi^{-}, \eta^{\prime} \pi^{-}$and $\rho^{0} \pi^{-}$-systems, nevertheless there is still room for a non-resonant interpretation of the phenomena [11, 12, 13, 14], and a more accurate measurement in a wider range of final state masses is needed.

A special kind of soft pion-nucleus interaction process is the inverse Compton scattering, or Primakoff scattering, in which the virtual photon exchanged between the beam pion and the electromagnetic field of the target nucleus emerges as a real photon of high energy in the final state. The measurement of the angular distribution of the $\pi \gamma$ Compton scattering cross-section allows to experimentally determine the electric $\left(\alpha_{\pi}\right)$ and magnetic $\left(\beta_{\pi}\right)$ polarizabilities. From the theoretical point of view, the values of $\alpha_{\pi}$ and $\beta_{\pi}$ have been calculated in the framework of the Chiral Perturbation Theory $(\chi \mathrm{PT})$ using information from radiative pion beta decays, predicting $\overline{\alpha_{\pi}}=(2.4 \pm 0.5) \cdot 10^{-4} \mathrm{fm}^{3}$ and $\overline{\beta_{\pi}}=(-2.1 \pm 0.5) \cdot 10^{-4} \mathrm{fm}^{3}[15]$. The experimental determination of the pion polarizabilities has not reached so far a comparable level of accuracy, and big discrepancies still exist between results obtained from different mechanisms[18].

\section{The COMPASS apparatus in the 2004 Pilot Run}

During the year 2004 COMPASS has performed a first run with hadron beams, with the aim of studying the Primakoff scattering and the diffractive meson production. In this section only the parts of the COMPASS apparatus relevant to this Pilot Run are described; for a complete overview of the experiment the reader is referred to [16] and references therein.

Negative pions of $190 \mathrm{GeV}$ of energy have been focused on thin nuclear targets of about 0.5 radiation lengths, shaped as cylinders with a diameter of $3 \mathrm{~cm}$. The typical $Z^{2}$ dependence of the Primakoff cross-section[15] (here $Z$ denotes the atomic number) allows to enhance the electromagnetic scattering process over the diffractive strong interaction by using heavy target materials. In COMPASS we decided to use lead for the Primakoff measurement, and we collected a small statistics with copper and carbon targets to verify the scaling behavior of the measured cross-section.

The precise measurement of the pion scattering angle is provided by two silicon micro-strip telescopes placed at a distance of $\sim 1 \mathrm{~m}$ before and after the target, each providing an angular resolution better than $20 \mu \mathrm{rad}$. The $\gamma$ energy and angle is measured by means of a GAMStype electromagnetic calorimeter (ECAL2), located at about $35 \mathrm{~m}$ downstream of the target. The calorimeter provides and energy resolution of $\frac{\sigma_{E}}{E}=1.5 \%+\frac{5.5 \%}{\sqrt{E(\mathrm{GeV})}}$ and a spatial resolution of 
$\sigma_{x, y}=\frac{6 \mathrm{~mm}}{\sqrt{E(\mathrm{GeV})}}$. The corresponding resolution on the four momentum transferred in the reaction $(t)$ is $\delta t \simeq 5 \cdot 10^{-4} \mathrm{GeV}^{2} / \mathrm{c}^{2}$.

The coincidence of two small scintillators located upstream of the target was used to select beam particles pointing to the target material (BeamTrigger). A hodoscope placed $\sim 35 \mathrm{~m}$ downstream of the target, with an acceptance between $20 \mathrm{GeV} / \mathrm{c}$ and $110 \mathrm{GeV} / \mathrm{c}$ in momentum, detected the scattered pions. Three small scintillators (BeamKillers) placed on the beam trajectory between the SM2 magnet and the ECAL2 calorimeter contributed to reject events without interactions in the target. Events with high energy photons or electromagnetic decays of neutral particles were selected by applying a minimum threshold on the summed energy of the ECAL2 calorimeter.

The selection of Primakoff scattering events was based on the coincidence of the BeamTrigger, of the scattered pion hodoscope and of a minimum energy deposit of about $50 \mathrm{GeV}$ in ECAL2.

A significant statistics of Primakoff scattering events has been also collected with a beam of $190 \mathrm{GeV}$ muons on lead. From the point of view of the electromagnetic scattering, muons behave approximately like point-like pions, thus allowing to directly measure the response of the apparatus to a point-like particle. Therefore, the measurement with a muon beam allows to accurately estimate the systematics errors affecting $\alpha_{\pi}$ and $\beta_{\pi}$.

In parallel to the polarizabilities measurement, data has been also collected for the diffractive $\eta$-meson production $\left(\pi^{-} Z \rightarrow \pi^{-} \eta Z \rightarrow \pi^{-}\left(\pi^{+} \pi^{-} \pi^{0}\right) Z\right.$ ), using all the available targets. The $\eta \rightarrow \pi^{+} \pi^{-} \pi^{0}$ decay mode is selected by requiring that at least two charged particles hit a scintillation counter located between the VetoBox and the downstream silicon telescope. Monte Carlo simulations of the reaction chain ensure that this solution does not introduce phase-space distortions in the detection of the $\pi^{-} \eta$ decay. The signal of the multiplicity counter is combined with the BeamTrigger and put in anti-coincidence with the BeamKillers to form the diffractive trigger.

The excellent resolution of the COMPASS spectrometer gives the opportunity to investigate hybrid mesons produced not only in the diffractive pion scattering, but also in photoproduction. Appropriate cuts on the four momentum transfer, as well as using targets of different atomic number, allow to approximately separate the two processes in the data analysis. This complementary information can improve our understanding of the underlying hybrid mesons production mechanism[17].

\section{Results}

In this section, the preliminary results of the analysis of a sub-sample of the data collected during the 2004 Pilot Run are presented. The data sample, corresponding to about $1 \%$ of the total available statistics, has been processed with the standard COMPASS event reconstruction software[19]. The interaction vertex is determined from the fit of the upstream and downstream track segments with a resolution of about $5 \mathrm{~mm}$ along the beam direction ( $z$-axis). The distribution of the reconstructed $z$ position of the interaction vertices is shown in Fig. 1-left. The peak corresponding to the interactions in the target is clearly visible at $z \simeq-310 \mathrm{~cm}$, and is well separated from the background interactions in the detectors surrounding the target. For the events of interest the four momentum transferred to the target nucleus is negligible. Therefore only events for which $\left|E_{\text {beam }}-\left(E_{\pi}+E_{\gamma}\right)<5\right| \mathrm{GeV}$ are kept for the following analysis steps. 

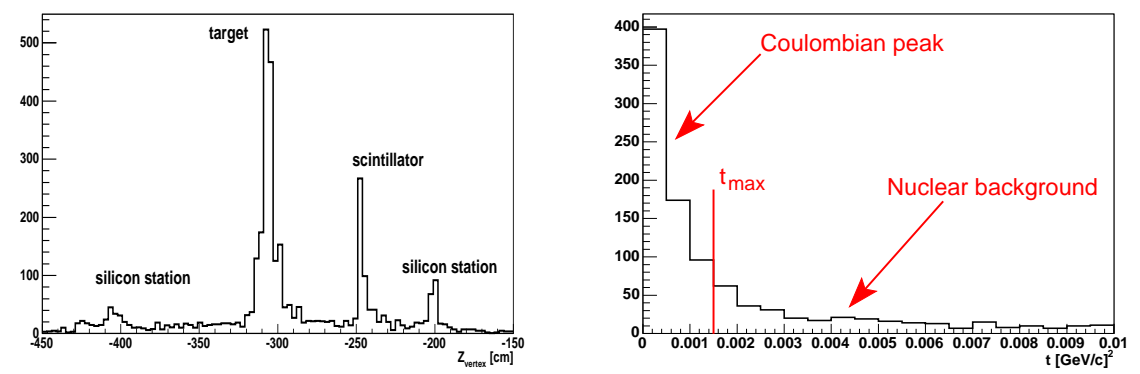

Figure 1: Preliminary results of the analysis of about $1 \%$ of Primakoff data. Left plot shows the reconstructed position of the interaction vertex along the beam direction ( $z$-axis), right plot shows the distribution of the reconstructed $t$-variable. Only events below $t_{\max }=1.5 \cdot 10^{-3} \mathrm{GeV}^{2} / \mathrm{c}^{2}$ are used in the Primakoff analysis.

The distribution of the reconstructed $t$ variable is shown in Fig.1-right; the Primakoff scattering events correspond to the region of $t<1.5 \cdot 10^{-3}$. The contribution of the diffractive scattering is evaluated from the extrapolation of the linear fit to the region of $t>2.0 \cdot 10^{-3}$.

The expected statistics of Primakoff scattering events on the lead target is of the order of 30000 events, approximately 4 times larger than the previous measurement at Serpukov [20].

\section{Central production of exotics}

Hybrid mesons are preferentially produced in periferal collisions. On the contrary, states composed mainly of gluons (glueballs) are preferentially produced in gluon-rich processes, like the pomeron-pomeron scattering in central proton-proton collisions (see for example [21], and references therein, for a review on the subject). The study of central production completes, together with the diffractive and Coulomb scattering, the search for exotic states in COMPASS, but requires a certain number of modifications to the COMPASS muon setup, that are briefly outlined in this section.

A beam of protons of $280 \mathrm{GeV}$ of energy will be focused on a liquid hydrogen $\left(\mathrm{LH}_{2}\right)$ target of $40 \mathrm{~cm}$ length and $3 \mathrm{~cm}$ diameter. The target material is inserted in the inner hole of a barrel-shaped time of flight (TOF) system, that is used to trigger on events in which the target proton recoils at large laboratory angles, and to measure the recoil energy. The larger beam energy compared to the muon case requires the re-alignment of the trackers downstream of the second magnet to compensate the smaller deviation by the magnetic fields. In addition, an efficient detection of the $\eta \eta$-system requires an upgrade of the existing electromagnetic calorimetry. The installation of the first electromagnetic calorimeter (ECAL1) will increase the $\gamma$ acceptance up to $150 \mathrm{mrad}$, matching that of the $\mathrm{LH}_{2}$ target system. The resulting acceptance for the $\eta \eta$-system is about $25 \%$ and is with good approximation constant over the full accessible mass range of $1.2 \div 4 \mathrm{GeV} / \mathrm{c}^{2}$, a fundamental requirement for the accurate partial wave analysis of the final state.

At a beam rate of $2 \cdot 10^{7}$ protons/SPS spill we expect about 30 events/hour for the lightest glueball candidate $\left(J^{P C}=0^{++}\right)$, predicted in the mass range $1.45 \div 1.75 \mathrm{GeV} / \mathrm{c}^{2}$. Due to the large 
acceptance and good particle identification properties of the COMPASS apparatus other channels like $\eta^{\prime} \eta$ or $K K$ can be investigated as well, in addition to the $\eta \eta$ decay mode.

\section{References}

[1] T. Barnes, Photoproduction of Hybrid Mesons, Cont. to Confinement III, Newport News, VA, June 1998, nucl-th/9907020

[2] N. Isgur, Phys.Rev. D60 (1999) 114016.

[3] F. Close, P.Page, Nucl. Phys. B443 (1995) 233.

[4] S.U. Chung et al.: Phys. Rev. D60 (1999) 092001.

[5] A. Abele et al.: Phys. Lett. B423 (1998) 175.

[6] A. Abele et al.: Phys. Lett. B446 (1999) 349.

[7] Y. Khokhlov et al.: Nucl. Phys. A663 (2000) 596.

[8] E.I. Ivanov et al.: Phys. Rev. Lett. 85 (2001) 3977.

[9] J. Kuhn et al.: e-Print archive hep-ex/0401004

[10] A.V. Popov et al.: in Proceedings of the 9th International Conference on Hadron Spectroscopy (Hadron 2001), AIP Conf. Proc. 619 (2002) 135.

[11] V. Dorofeev et al.: Proceeding of the 9th International Conference on Hadron Spectroscopy (Hadron 2001), AIP Conf. Proc. 619 (2002) 143.

[12] A.P. Szczepaniak, M. Swat, A.R. Dzierba, S. Teige: Phys. Rev. Lett 91 (2003) 092002.

[13] A. Donnachie, R. Philip, P.R. Page: Phys. Rev. D58 (1998) 114012.

[14] A.R. Dzierba et al.: Phys. Rev. D67 (2003) 094015.

[15] U. Bürgi, Phys. Lett. B377 (1996) 147.

[16] G.K. Mallot, Nucl. Instrum. Meth. A518 (2004) 121; F. Bradamante, hep-ex/0411076, and references therein.

[17] M. Moinester, http://arxiv.org/abs/hep-ex/0301023, Proc. of COMPASS Future Physics Workshop, http://pccosrv1.cern.ch/compass/publications/2004_yellow/

[18] M. Colantoni et al., Czech. J. Phys. 55 Suppl. A (2005) A35, and references therein.

[19] Compass Reconstruction and Analysis Software, http: / / coral.cern.ch/

[20] Y.M. Antipov et al.: Phys. Lett B121 (1983) 445.

[21] W. Dünnweber, Proc. of the Workshop on Future Physics COMPASS, CERN Yellow Report CERN-2004-011; S.V. Donskov, Proc. of the Workshop on Future Physics COMPASS, CERN Yellow Report CERN-2004-011. 